\title{
Fixing Device for a Planar Object by Incorporating Jamming Transition Phenomenon and a Suction Unit
}

\author{
Akira Bekku and Yoshikazu Nakajima \\ Department of Bioengineering, School of Engineering, The University of Tokyo, Hongo 7-3-1, Bunkyo, Tokyo 113-8656, Japan \\ Correspondence should be addressed to Akira Bekku; bekku@image.t.u-tokyo.ac.jp
}

Received 12 December 2014; Revised 14 April 2015; Accepted 22 April 2015

Academic Editor: Giovanni Muscato

Copyright (C) 2015 A. Bekku and Y. Nakajima. This is an open access article distributed under the Creative Commons Attribution License, which permits unrestricted use, distribution, and reproduction in any medium, provided the original work is properly cited.

\begin{abstract}
This paper presents a versatile method to install a small machining robot onto a planar or slightly curved object. Normally, gripping tools provide a rigid connection between a robot and a target. However, if there is no graspable area on the target, the gripper cannot perform well. To overcome this problem, we proposed a deformable device that works as a universal suction pad and that can hold an ungraspable target by utilizing the jamming transition phenomenon. We determined the effect of the type of grains on the jamming transition phenomenon in terms of stability under jammed conditions. By comparing the four types of grains, we found that the tetrahedral-shaped grains performed the best. Subsequently, we evaluated the performance of the device. The suction force was $166-\mathrm{N}$ when the proposed device was affixed to a curved target. Next, we evaluated the stiffness of the device. The average deformations of the device were $0.10 \mathrm{~mm}, 0.35 \mathrm{~mm}$, and $0.04 \mathrm{~mm}$, respectively, when $50-\mathrm{N}$ downward vertical, horizontal, and upward vertical directional loads were applied to the device, which was affixed to the curved target. The results suggest that the proposed device performed well in affixing a robot onto a target.
\end{abstract}

\section{Introduction}

The goal of this research is to develop a versatile fixing device for a planar or smooth object without physical invasiveness and electromagnetic interference. The type of fixing method is important for connecting a fixing unit and a target object. For a small machining robotic system, this task is mainly used to mount the system onto a target. The importance of affixing a small robot onto a target is high because the movement of the robot decreases the fixing stability. Thus, fixing methods for portable robotic systems are widely studied. Body-mounted surgical robot systems and wallclimbing robot systems are examples of these. Their common characteristics are described in the following sections. A fixing surface is planar or has a slightly curved shape and has few graspable areas. Anchoring is not adequate for a target because it can damage the target.

Fixing methods can be classified by the source of the fixing force. Pressing force is commonly used to connect two objects. A screw is a typical tool that forces an object against another object that has a tapped hole. Fixed objects are compressively stressed, and a screw is subjected to tensile stress. The repulsion of both stresses and an associated friction force works to maintain rigid fixation. However, as noted previously, we can use a screw only when a fixing target has a tapped hole, and this condition is a special case. Thus, this method is difficult to use for small robot fixation.

A grip force-generated by a tool such as a robot hand or clamper-generates contact force by pinching the target from both sides. The normal directional force, tangential directional force, and friction force are at equilibrium when the tool is holding a target and it interlocks with an object. Multijoint hand manipulation $[1,2]$ is conventionally introduced to hold an object without physical invasiveness. This method can hold a target that has no tapped hole without crushing the target.

A strap uses pressing force too and is used in some bodymounted robots $[3,4]$. To make the tensile force act as a compressive force, the fixing target must be enclosed by a closed loop of a strap. The deformability of the strap is not adequate for rigid fixation because a long, thin object can cause instability. 
A suction unit utilizes air pressure as the pressing force for fixation, and many wall-climbing robots $[5,6]$ use this function to cling onto a wall. In addition, this type of unit can stick to soft material such as body organ. A stabilizer is used to immobilize a particular vessel of heart during an offpump coronary artery bypass operation [7]. The suction force is effective to fix a breakable or soft target by controlling the amount of negative pressure. Normal directional movement is prevented by the reaction force from the standing surface and air pressure. Tangential directional movement is prevented by the friction force. The fixing performance depends on the amount of negative pressure inside the suction unit and the atmospheric pressure outside the unit. An important feature of this method is that it can hold a planar object without interlock. A heavy unit can utilize its own weight. The gravitational force, normal directional reactive force, and friction force are large enough to stabilize a heavy object. However, the normal direction of the fixing surface must be upward in a vertical direction.

Attracting force is another source of a fixing force, and adhesive tape is a typical tool. When adhesive material fills a molecular-scale gap, two objects adhere to each other. An adhesive material connects two objects by intermolecular force. For the aforementioned reasons, a suction unit is best for small robot fixation.

The number of fixing points is another factor to consider. The stability of fixation is limited with more than three contact points. To obtain more than a three-point contact, the position and rotation of the sucking unit must be controlled. A bobbing head or bellows suction unit can correspond to a certain level of inclination. However, the tolerance range of concavity and convexity is not as large. For practical purposes, the base of the suction unit must correspond to the shape of the target surface. A tailor-made template can be created based on an individual target; this is a straightforward device. In clinical cases, a template of a patient's bone frame has been used for lumber puncture surgery [8]. The disadvantages of this method are that the tailor-made template must be fabricated before beginning the operation, and it lacks versatility. Therefore, a flexible holding device that contours a target surface is ideal from a practical perspective. To achieve a versatile variable device that freely functions like a human hand, a multifingered robot hand is beneficial. However, in addition to the mechanical design of the robot hand, the tactile and visual sense of the robot hand system is also an important factor to achieve successful gripping without breaking the target. The feedback control algorithm and actuator operation tend to become complex. Brown et al. [9] focused on the simple idea of attaching an elastic bag filled with granular matter and showed that the gripping process is controlled by the jamming transition phenomenon [10]. The jamming transition phenomenon is the phase transition of the grain aggregate. The phase changes between a fluid and a solid. The state of the grain aggregate transitions from a fluid-like state to a solid-like state when the density of the grains increases. The grain aggregate returns to a fluid-like state when the density of the grains reduces. The process of holding is described in the following sections. Initially, the "universal gripper" has a fluid-like state and conforms to the surface of a target. Then, the gripper transitions to a solidlike state by evacuating the gas inside the elastic bag and holding the target. When the gripper becomes a rigid mold of the target, the gripper can lift the target well. The advantage of this method is that the gripper needs no force sensing because the granular matters are autonomously displaced from the contacting area without damaging the target. The performance of the universal gripper was evaluated at the point of holding and lifting performance. When the base of a suction unit is fabricated by this mechanism, the suction unit can correspond to a target with various shapes.

In this study, we analyzed the stationary condition of jammed grains and proposed an effective shape of grains to achieve a highly rigid solid state of the fixing device that uses the jamming transition phenomenon. We have demonstrated possibility of this type of novel fixing device before [11]. In previous work, we combined the fixing device with the surgical assistance robot for spine puncture surgery. We evaluated the stability of the fixing device at the condition of needle puncture operation on the plastic human back phantom. However, there still are other unclear points in establishing the versatile fixing device. We use the jamming transition phenomenon to control the solid phase and fluid phase of the fixing device. But the behavior of grains at both conditions is not evaluated in the previous work. To optimize the proposed fixing devise, analysis and optimization of grain at phase transition must be done.

In addition, the stiffness of the fixing device is not evaluated quantitatively and the stiffness characteristic has to be evaluated to determine the effective range and limitation of the device. By using a fixing device filled with characteristically shaped grains, we implemented a fixing device and evaluated the fixing performance on a planar and slightly curved object.

\section{Method}

The proposed fixing device utilizes the phase transition of high-density grains, and this phenomenon is called the jamming transition phenomenon. We use this phenomenon to mold a fixing device that can hold a target in real time. The fixing device solves the trade-off problem between the level of damage caused and the rigidity of the device. The conceptual drawing of the proposed device is shown in Figure 1(a). The shape of the device is the same as the vacuum pad. A normal vacuum pad is made of rubber. However, our device is made of an elastic container and packed grains. Thus, the device is deformed by the outside pressure and contours the holding object that is pressed against the device. When the air inside the device is evacuated from the suction port to cause the jamming transition phenomenon, the grains are compressed on the inside, and the density of the grains increases. Subsequently, the jamming transition phenomenon occurs, and the device transitions from a fluid-like state to a solidlike state. The process of fixation is shown in Figure 1(b). First, we prepared the device above the target. The device contained fluid-like grains and deformed easily. The grains are evenly distributed and the container is of indefinite shape. The container is in a reactive mode. Second, we pressed 


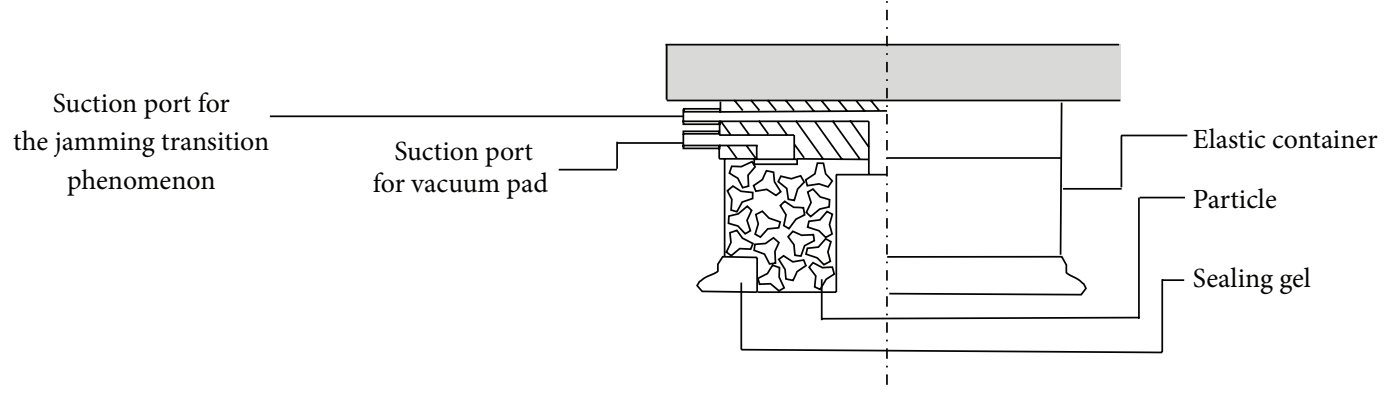

(a)

Air pressure values are almost the same and the density of particles is low

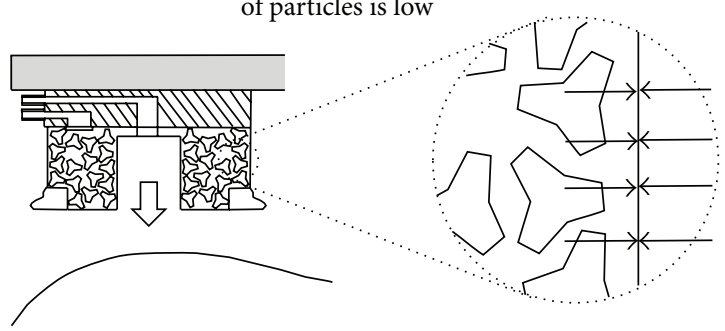

(A) Deformable state

The air pressure inside the fixing pad is reduced, and the density is increased

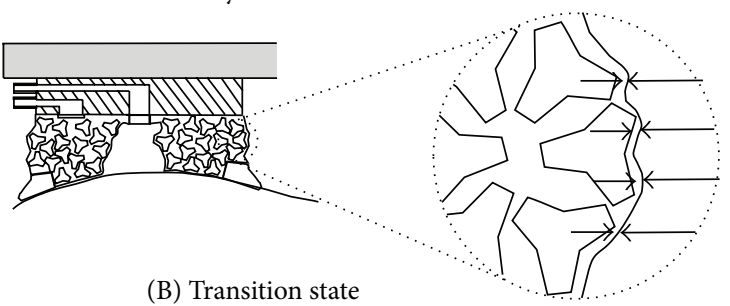

(b)

FIGURE 1: Process diagram of medical device affixation onto patient. (a) Half-section drawing of vacuum pad of fixing device. (b) Process diagram of fixation with proposed fixing device. (A) The fixing device freely deforms when the device is pressed against the object. (B) The fixing device contours to the patient's body and transitions to a solid state by vacuuming air. (C) The vacuum pad of the solid fixing device sticks to the patient's body.

the device against the target and started vacuuming from the suction ports. The required suppression strength depends on the flow property of the grains inside of the container. At the fluid-like condition, when the container is suppressed onto the object, the grains are pushed out to a vacant space and the contour of container changes. If the fixing device is suppressed successfully onto the target, the fixing device holds the target without any space. The phase transition of the grains started when the device remained in close contact with the target and the vacuum pad became functional. The final outer shape of the fixing device is determined by the air pressure differential that is made by the suction unit. In this paper, the absolute amount of suction pressure was $>20 \mathrm{kPa}$ that is enough to change the shape of the fixing device ideally. Finally, the device that finished contouring to the surface of the target hardened. The design of the device enabled it to be strongly affixed to the surface of the target by the suction force. The vertical displacement of the fixing device is prevented by the air pressure and the horizontal displacement is blocked by the friction force between the fixing device and the target.

The resolution of contouring is ensured by the granularity and flowability of the grains, and the stiffness of the grain aggregate depends on its ease of being jammed. We optimized the parameters of the grains and the design of the device.

\section{Grain Parameters for Jamming Transition}

Stiffness is one of the most important parameters of the fixing device because this parameter is crucial in maintaining the geometrical relationship between the target and the fixing device. The stiffness of the device depends on the physical 


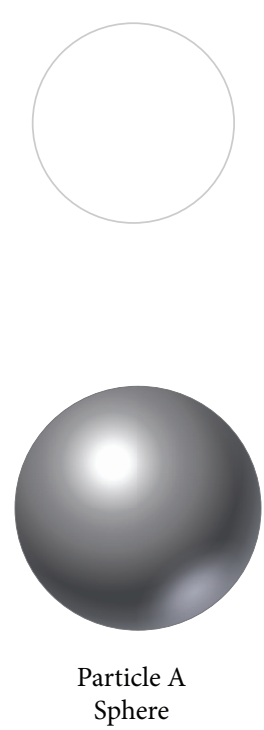

Sphere
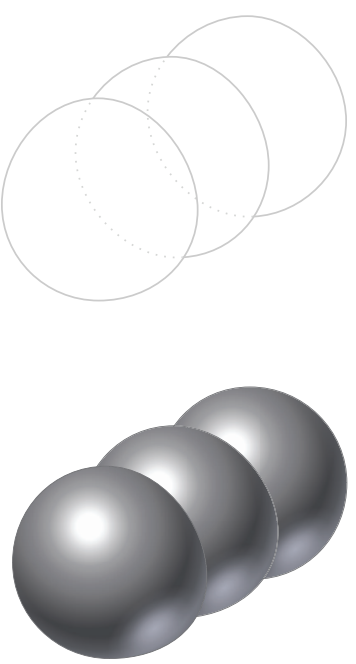

Particle B

Rod

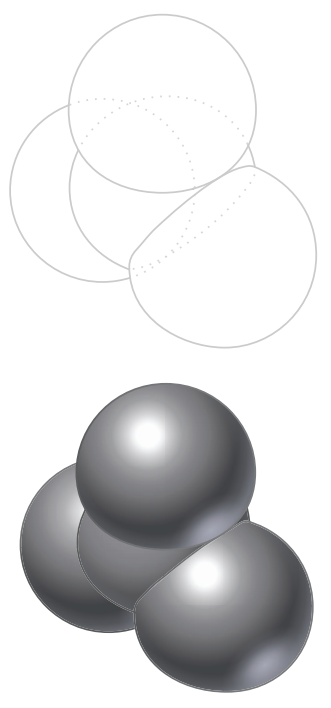

Particle C Triangle

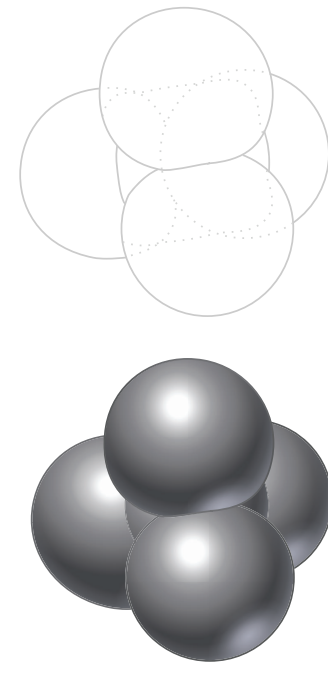

Particle D Tetra

FIgURE 2: Types of simulated grains.

property of the grain and the collapse behavior of the jammed grain aggregate because the deformation of the device is due to the deformation, collapse, and rearrangement of the grains.

The relationship between the behavior and properties of the grain aggregates is studied in terms of the grain shape. Miskin and Jaeger [12] researched the optimized design of grains by using evolutionary computing that enables the systematic analysis of the effect of grain shape. They evaluated the stress-strain relationship by using a compression machine with grains that were fabricated using a three-dimensional (3D) printer. However, the relationship between grain shape and the stiffness of the jammed aggregate has still not been systematized.

The jamming transition phenomenon is caused by the interaction of nearby grains. Therefore, the geometrical restriction is an important factor in grain design. When grains are restricted by their shape and the position of neighboring grains, they prevent grain displacement from occurring in the initial condition.

\section{Experiment to Evaluate the Property of Grains}

We evaluated the contact state of grains in the grain simulations. To simulate the jammed condition of various-shaped grains, we charged four types of grains in a rectangular enclosure. The shape of the grains was set as shown in Figure 2. The shapes of the grains have different characteristics; thus, the rotation restriction caused by the physical interlock was different. In the simulation, the grains were reconstructed by spheres that were rigidly connected to each other. The movement of the grains was calculated by using the model of a passively moving solid [13]. The contact and collision model of the sphere element was based on Hertzian contact theory. The contacting force in the normal direction was calculated by (1) when the distortion amount in the normal direction is $\boldsymbol{\delta}_{n}$ and the grain velocity in the normal direction is $\mathbf{V}_{n}$. Equation (2) is a tangential force when the distortion amount and velocity in the tangential direction are $\boldsymbol{\delta}_{t}$ and $\mathbf{V}_{t}$. Under the condition that slip is generated between the contacting grains, the tangential force is described as (3). The radius of the sphere is $r(\mathrm{~mm})$, Young's modulus is $E$, Poisson's ratio is $\nu$, the viscosity coefficient is $\eta$, and the friction coefficient is $\mu$. Consider

$$
\begin{aligned}
& \mathbf{F}_{n}=-\frac{\sqrt{2 r} E}{3\left(1-v^{2}\right)} \boldsymbol{\delta}_{n}^{3 / 2}-\eta \mathbf{V}_{n}, \\
& \mathbf{F}_{t}=-\frac{2 \sqrt{r} E}{(1+\nu)(2-\nu)} \boldsymbol{\delta}_{t}-\eta \mathbf{V}_{t}, \\
& \mathbf{F}_{t}=-\mu\left|F_{n}\right| \frac{\boldsymbol{\delta}_{t}}{\left|\boldsymbol{\delta}_{t}\right|} .
\end{aligned}
$$

The viscosity coefficient $\eta$ was determined from the coefficient of restitution $e$ to satisfy $e=-\eta \pi / \sqrt{4 k^{2}-\eta} \cdot k$ is the spring coefficient that is described as $\sqrt{2 r} E / 3\left(1-v^{2}\right)$.

The movement of a passively moving solid was calculated under the condition that the relative configuration of the spherical grains was fixed. The size of the enclosure was 100 $\times 20 \times 20 \mathrm{~mm}^{3}$; the volume of a grain was $6.82 \mathrm{~mm}^{3}$. Other parameters are listed in Table 1. We charged 2500 grains and confirmed the contact condition and slipping tendency for each grain type. To reconstruct a well-jammed condition, the friction coefficient of grains was set to 0 during grain charging. The grains were initially generated at a height of $17.5 \mathrm{~mm}$. The posture and lateral location were randomly selected. The contact condition was evaluated after all grains were generated and the arrangement of grains was stabilized under the force of gravity. 


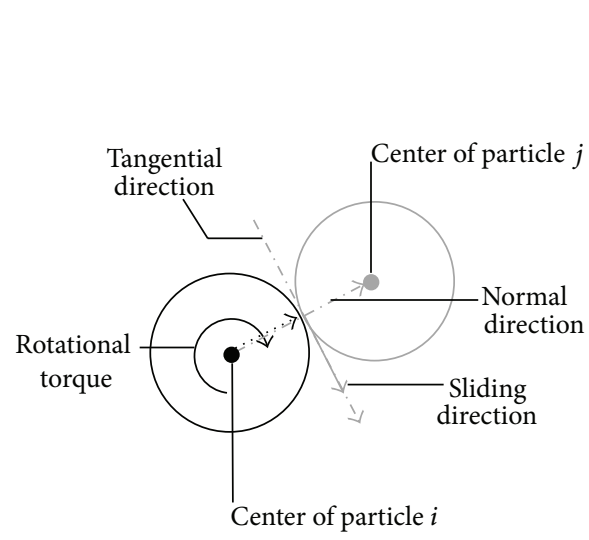

(a)

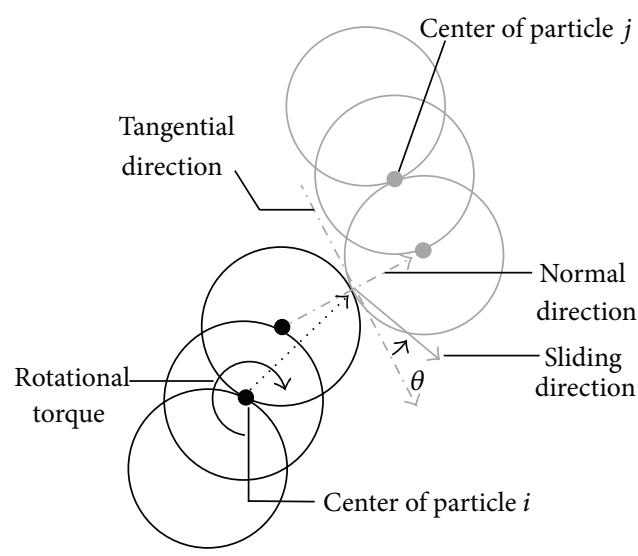

(b)

FIGURE 3: Contacting condition of nonspherical grains and contact angle $\theta$. (a) Contacting condition of spherical grain and (b) contacting condition of nonspherical grain.

TABLE 1: Physical parameters for simulation experiment.

\begin{tabular}{lc}
\hline Weight $(\mathrm{g})$ & $1.364 \times 10^{-2}$ \\
Volume $\left(\mathrm{mm}^{3}\right)$ & 6.82 \\
Young's modulus $(\mathrm{GPa})$ & 0.80 \\
Poisson's ratio & 0.5 \\
Reflection coefficient & 0.01 \\
\hline
\end{tabular}

We evaluated the contacting number of each grain under the same condition. In addition, the average contact force of a contact point was measured. The contact position was also evaluated to examine the engaged condition of grains. We then evaluated the condition of the grain's interlock inside the grain aggregates.

The differentiation between contact and noncontact states is based on the distance between two spheres that are contained in different grains. If the distance is smaller than the diameter of a sphere, the grains have made contact.

The contact position of the grains was evaluated for only Grains B, C, and D because Grain A is a sphere, and the contact ratio of all points on the grain surface is the same. Grain B freely rotates around the longitudinal axis. The contact probability of Grains $\mathrm{C}$ and $\mathrm{D}$ was described in $3 \mathrm{D}$ space. The contact ratio was calculated for each $0.01 \times 0.01 \times$ $0.01 \mathrm{~mm}^{3}$ area in $3 \mathrm{D}$ space.

The grains at jammed condition change into unjammed condition when shearing or slipping changes the configuration of grains. To break free from the jammed condition, the required force is the equilibrant force to the static friction force at the contact points. The force required to produce slipping of grains is determined by the friction coefficient and angle of contact with respect to shear direction (Figure 3). When the shape of grain is sphere and the normal force at contact point is $\mathbf{F}_{\mathbf{n}}$, the relationship between the tangential unit vector $\mathbf{t}$ and the tangential force $\mathbf{F}_{\mathbf{t}}$ is $\mathbf{F}_{\mathbf{t}}=\mu\left|\mathbf{F}_{\mathbf{n}}\right| \mathbf{t}$ and the slipping force $\mathbf{F}_{\mathbf{s}}=\mathbf{F}_{\mathbf{t}}$. However, if the shape of grain is not sphere and direction of shearing force is different from the tangential one, the required force to cause slipping increases.

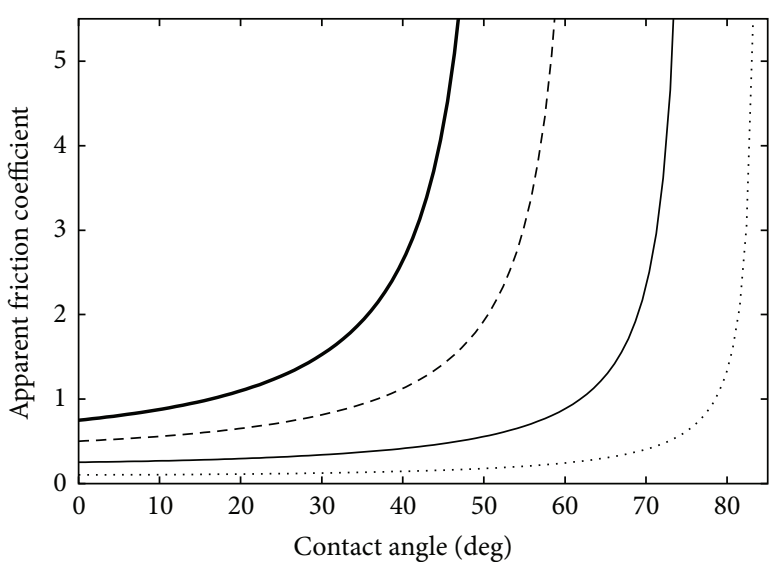

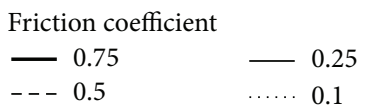

FIGURE 4: Relationship between the apparent friction coefficient and contact angle.

When the angular difference between the rotation vector $\mathbf{r}$ and the tangential direction is $\theta$, the slipping force $\mathbf{F}_{\mathbf{s}}$ is divided into normal direction and tangential direction. The norm of the normal force is $\left|\mathbf{F}_{\mathbf{s}}\right| \sin \theta$ and that of the tangential force is $\left|\mathbf{F}_{s}\right| \cos \theta$.

As a result, the required tangential force $F_{t}$ is $F_{t}=\mu\left|F_{n}\right| t$ as the sphere but the required force to cause slipping is $\mathbf{F}_{\mathbf{t}}=$ $\mu /(\cos \theta-\mu \sin \theta)\left|\mathbf{F}_{\mathbf{n}}\right| \mathbf{t}$.

The ratio of the slipping force of sphere grain and that of other conditions is $\mu: \mu /(\cos \theta-\mu \sin \theta)$. The relationship between the contact angle $\theta$ and the apparent friction coefficient $\mu /(\cos \theta-\mu \sin \theta)$ is shown as Figure 4 .

To evaluate the apparent friction coefficient of each type of grains, the apparent friction coefficient at the condition of slipping by rotation is calculated and listed up.

When the apparent friction coefficient $\mu^{\prime}=\mu /(\cos \theta-$ $\mu \sin \theta)$ is defined by the rotation direction and contacting 
TABLE 2: Contact angle and apparent friction coefficient of 4-type particles.

\begin{tabular}{|c|c|c|c|c|c|c|c|c|c|c|c|}
\hline \multicolumn{3}{|c|}{ Sphere } & \multicolumn{3}{|c|}{ Rod } & \multicolumn{3}{|c|}{ Triangle } & \multicolumn{3}{|c|}{ Tetra } \\
\hline $\begin{array}{l}\text { Contact } \\
\text { number }\end{array}$ & $\begin{array}{c}\text { Contact } \\
\text { angle }\end{array}$ & $\begin{array}{c}\text { Apparent } \\
\text { friction }\end{array}$ & $\begin{array}{l}\text { Contact } \\
\text { number }\end{array}$ & $\begin{array}{c}\text { Contact } \\
\text { angle }\end{array}$ & $\begin{array}{l}\text { Apparent } \\
\text { friction }\end{array}$ & $\begin{array}{l}\text { Contact } \\
\text { number }\end{array}$ & $\begin{array}{l}\text { Contact } \\
\text { angle }\end{array}$ & $\begin{array}{l}\text { Apparent } \\
\text { friction }\end{array}$ & $\begin{array}{l}\text { Contact } \\
\text { number }\end{array}$ & $\begin{array}{c}\text { Contact } \\
\text { angle }\end{array}$ & $\begin{array}{l}\text { Apparent } \\
\text { friction }\end{array}$ \\
\hline \multirow{4}{*}{4.58} & 0.00 & 0.10 & \multirow{4}{*}{7.85} & 19.25 & 0.11 & \multirow{4}{*}{5.45} & 20.80 & 0.11 & \multirow{4}{*}{9.94} & 20.78 & 0.11 \\
\hline & 0.00 & 0.25 & & 13.84 & 0.27 & & 16.72 & 0.28 & & 16.68 & 0.28 \\
\hline & 0.00 & 0.50 & & 8.85 & 0.55 & & 15.42 & 0.60 & & 15.16 & 0.60 \\
\hline & 0.00 & 0.75 & & 6.34 & 0.82 & & 20.22 & 1.10 & & 18.03 & 1.04 \\
\hline
\end{tabular}

condition, the required rotational torque $\mathbf{R}=\mu /(\cos \theta-$ $\mu \sin \theta)\left|\mathbf{F}_{\mathbf{n}}\right|(\mathbf{c} \times \mathbf{t})$ to cause slipping can be calculated by the apparent friction coefficient and the vector from the center of grains to the contact point $\mathbf{c}$. We simulated the required torque to rotate the grains in all directions for $360^{\circ}$ and evaluated the average torque and standard deviation that were required to rotate a grain. The rotation directions were divided into equally divided 1899 directions.

The torque is defined by the friction coefficient and the number of contacts with neighbors.

Not only the stiffness of grains at the solid-like state, but also fluidness of grains at the fluid-like state is important to discuss the performance of the fixing device that can contour the shape of human body. To evaluate the fluidness of the different grains that have specific friction coefficient, we evaluated the packing ratio of the grains that fell in the 30 $\times 30 \times 30 \mathrm{~mm}^{3}$ container under the grain's own weight. 800 tetrahedral-shaped grains were randomly generated in the container and the initial packing ratio is 0.20 . After the grains move downside and stand still, we measured the packing ratio of the grains. Because the grains fall and the grains are concentrated to the height of $10 \mathrm{~mm}$, we excepted the upper blank area of the container in this evaluation.

\section{Results of the Grain Property Experiments}

The contact number of each grain is shown in Figure 5. The contact number depends on the ease of packing and the contact number of one pair. Rod-shaped and tetrahedralshaped grain can reach the neighbor grain. However, the triangle-shaped grain is difficult to achieve tight packing. Thus, there is significant difference among the particles.

When the contact number is large, it implies that the contacting force is dispersed and the risks of compression fragmentation decrease (Figure 6). The contacting number and apparent friction coefficient are listed in Table 2. The contact angles were calculated by apparent friction coefficient. With the number of contacts and apparent frictional force, the stiffness of the jammed grain is defined. The contact ratio on the grain surface that relates the contact angle is shown in Figure 7. It presents the condition of the interlock. The shape of grain defines the position of interlock, the contact angle, and the inaccessible surface. Almost every position on the face of a grain has equal probability of contacting. However, the surface point with a significantly high probability of contacting is constricted part of grain. It shows that nonspherical grains interdigitate geometrically at shape-specific position. When the head of a convex superior portion comes into

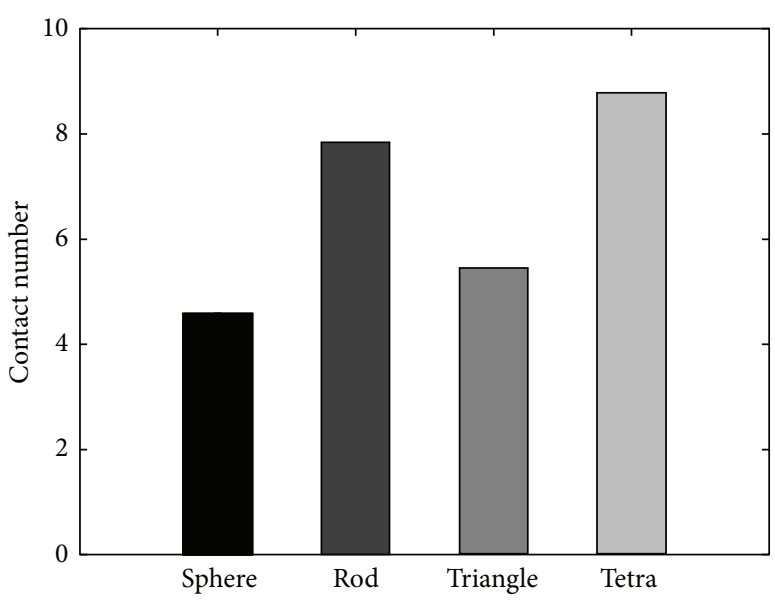

FIGURE 5: Average number of contacts.

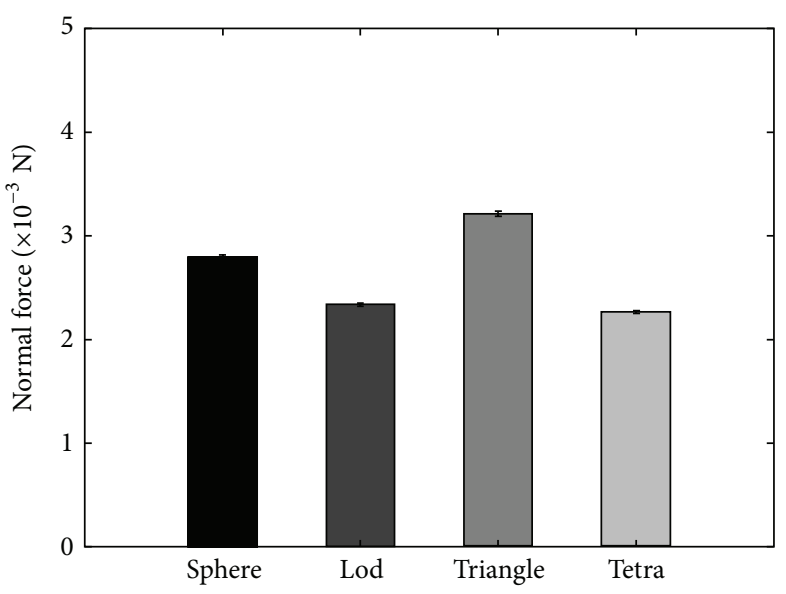

FIGURE 6: Contact force in normal direction.

contact with neighbor grains at a high rate, displacement in the tangential direction tends to occur. Considerations that should be entertained as causes of the bias include the singular point that produces stably positioned grains. But the constricted part of the rod-shaped grains is due to the approximate model that is designed by the rigid-connected spheres. If the jointing part is smooth, it is expected that the distribution of contacting position changes and the apparent friction coefficient shows different value. 

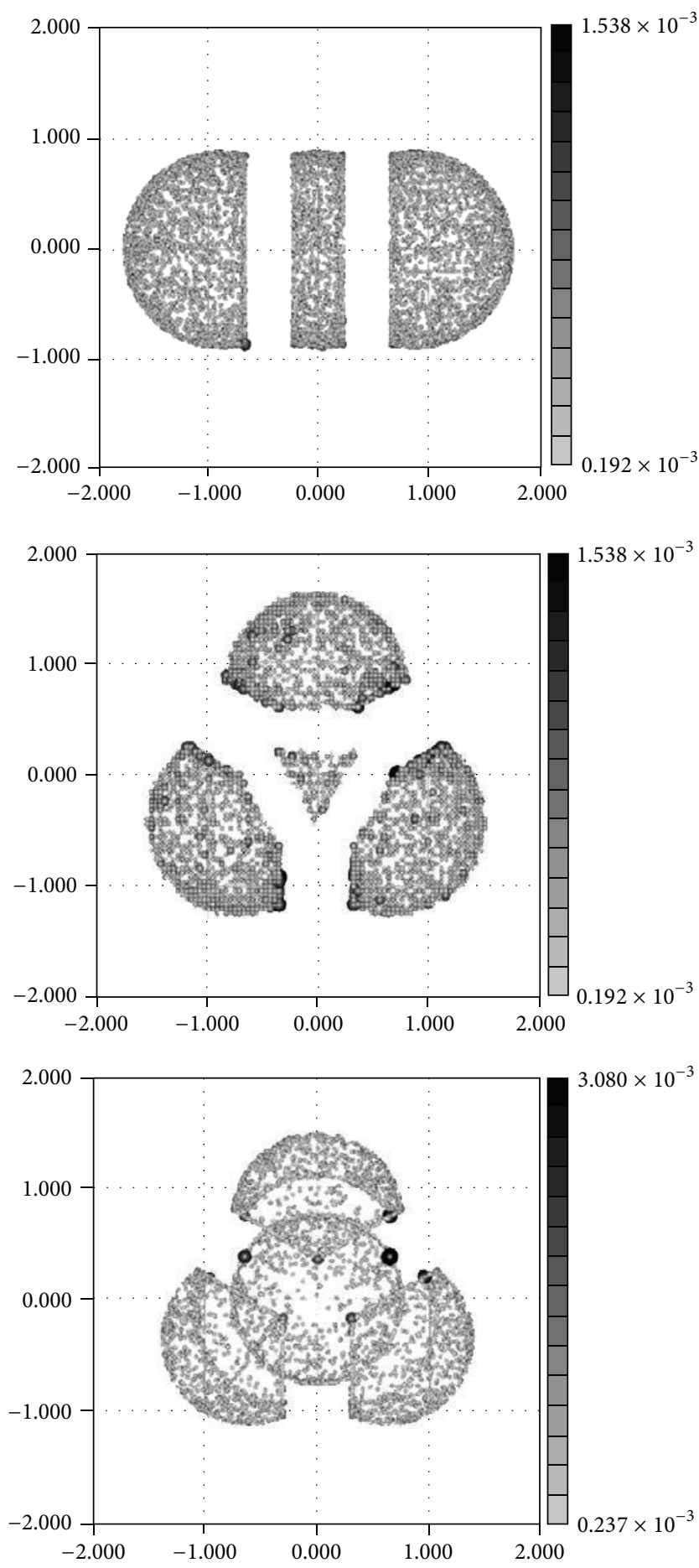

Figure 7: Contact ratio on the grain surface.

The effect of the contact position distribution results in the rotation restriction and the norm of the rotational torque $|\mathbf{R}|=\mu /(\cos \theta-\mu \sin \theta)\left|\mathbf{F}_{\mathbf{n}}\right||\mathbf{c}|$ is shown in Figure 8 .

The figure shows that tetrahedral-shaped grains require high torque to generate slip between grains when the friction coefficient is less than 0.5 . When the friction coefficient is 0.75 , rod-shaped grains are well jammed and prevent rotational slipping. Thus, the optimized shape for the fixing device

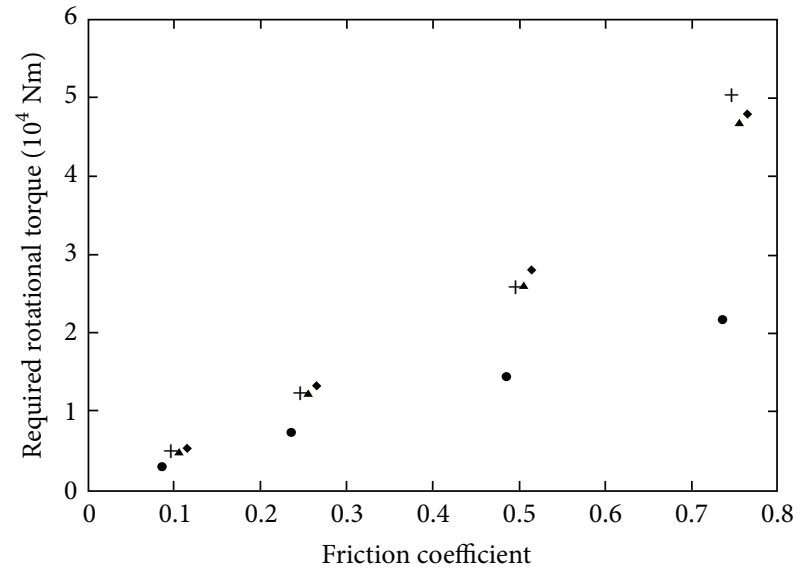

Shape of grain

- Sphere

$+\operatorname{Rod}$

\ Triangle

- Tetra

FIGURE 8: Rotation torque required for intergrain slipping.

TABLE 3: Packing ratio of the tetrahedral-shaped grains.

\begin{tabular}{lllll}
\hline Friction coefficient & 0.10 & 0.25 & 0.50 & 0.75 \\
\hline Packing ratio & 0.59 & 0.59 & 0.61 & 0.62 \\
\hline
\end{tabular}

depends on the friction coefficient of the grain material. As the high frictional material, glass, silver, and copper are included. However, as the grains for our device, a hardened and tough material such as ceramic is adequate. Zirconia is a leading example of ceramic. The friction coefficient of Zirconia is about 0.4. For our purposes, the tetrahedralshaped grains are appropriate when the friction coefficient of grains is less than that of Zirconia. The tetrahedralshaped grains will constrain the nearby grains and prevent the structural change of the jammed grain aggregate. The tetrahedral-shaped grains work well among the evaluated grains.

The packing ratios of the tetrahedral-shaped grains are listed in Table 3. It shows that the difference of fluidness is not so large and frictional coefficient does not have tight restriction.

\section{Implementation of Holding Device}

The holding device needs to contour the individual shape of the target to cling to it and remove any empty space that could potentially cause gaps. Then, the device needs to harden while maintaining this shape and remaining strongly attached to the target surface. For our purposes, the jamming transition phenomenon and a sucking system were selected for the optimized device. To hold the entire area around the affected area, the device has to have enough initial thickness as a deformation margin. The arrangement of the fixed point and the size of the device were determined as shown in Figure 9. We designed a tripod device that avoids large irregularity while the device is mounted onto a target. The height of the suction pad is $20 \mathrm{~mm}$, and this is the deformation margin. 


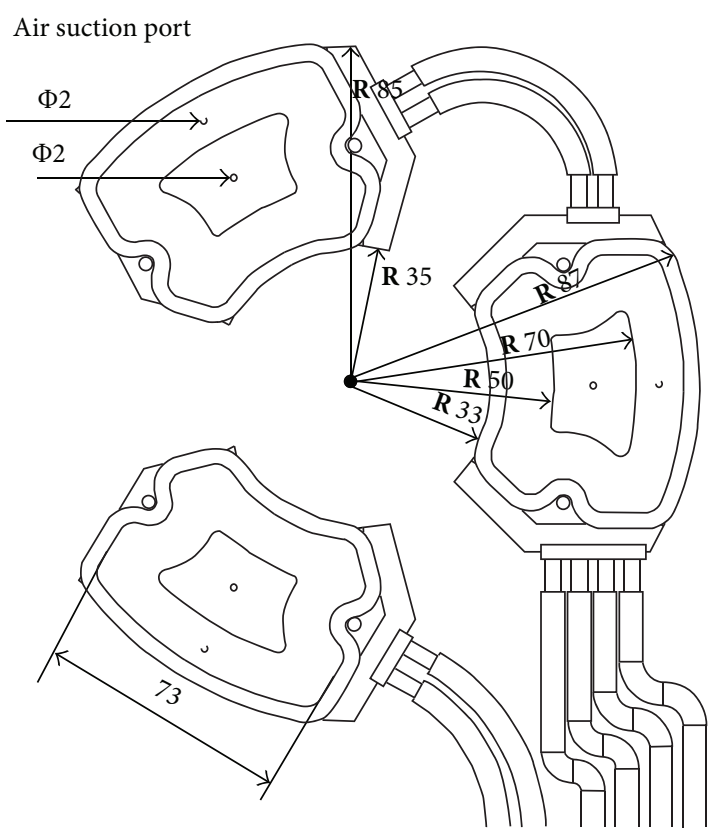

Unit: $\mathrm{mm}$

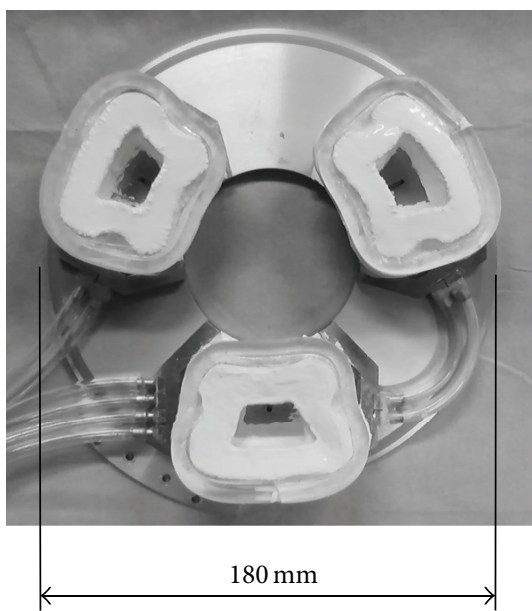

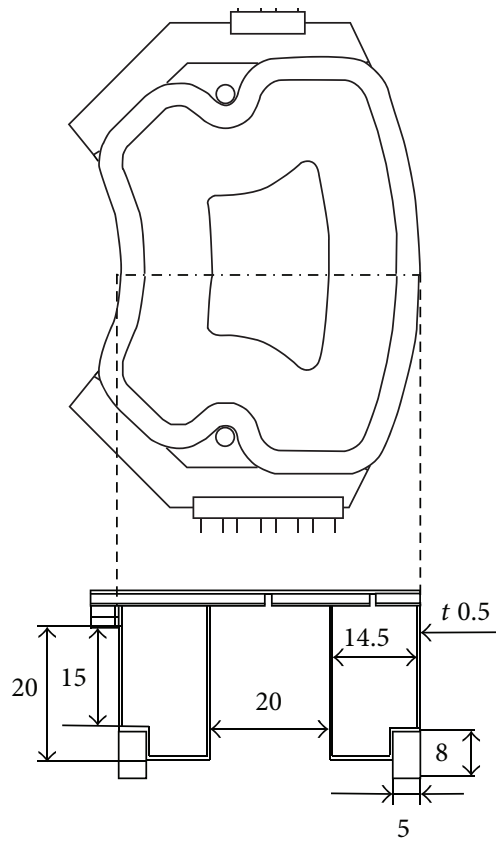
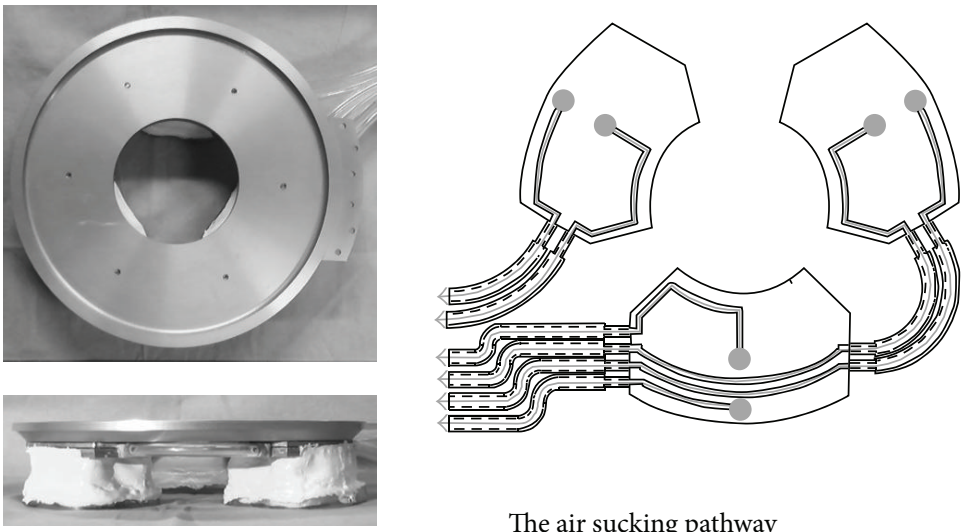

The air sucking pathway inside the fixing device

FIgURE 9: Jamming holding device and pathway of the air flow for the suction system.

The sectional area of the device has an implication for the displacement of the device. The area that comes into contact with the target was designed to be $5.2 \times 10^{3} \mathrm{~mm}^{2}$ large. The inner area of the suction pad determines the suction force. When the vacuum pressure in the suction pad is $P(\mathrm{MPa})$ and the inner area of the suction pad is $S\left(\mathrm{~mm}^{2}\right)$, the suction force $W(\mathrm{~N})$ is given by

$$
W=P S .
$$

The inner area of the suction pad is within the sealing material. We designed this area to be $7.0 \times 10^{3} \mathrm{~mm}^{2}$.

The vacuum system was composed of an air compressor (CP-100, Nakatomi Co., Ltd.) and a vacuum generator (VBH07-66P, Pisco Co., Ltd.). In this setup, we set the negative pressure in the suction pad at $-30 \mathrm{kPa}$ (Figure 10). To control the vacuum pressure in the suction pad, a vacuum regulator (RVV6UG, Pisco Co., Ltd.) was connected between the vacuum generator and the vacuum ports inside the suction pad.

The container that encapsulates the grains should be elastic enough to contour a target. However, an elastic material can easily be deformed by an external force. To prevent large displacement of the holding device, the thickness of the container should be minimized. We fabricated the elastic container with a $0.5-\mathrm{mm}$ thick silicon rubber cloth. The compressive elastic modulus of the silicon rubber (KE-12, Shin-Etsu Chemical Co., Ltd.) is $0.3 \mathrm{MPa}$. The sealing gel that surrounded the external frame of the suction pad was a nonviscous gel sealant (CRG-N0505, TANAC Co., Ltd.) that has a degree of extensibility over $1500 \%$. The grain is gypseous material (zp150, 3D Systems, Inc.) and it is fabricated by the 3D printer (ZPrinter 450, 3D Systems, Inc.). The accuracy of output is $0.3 \mathrm{~mm}$. 


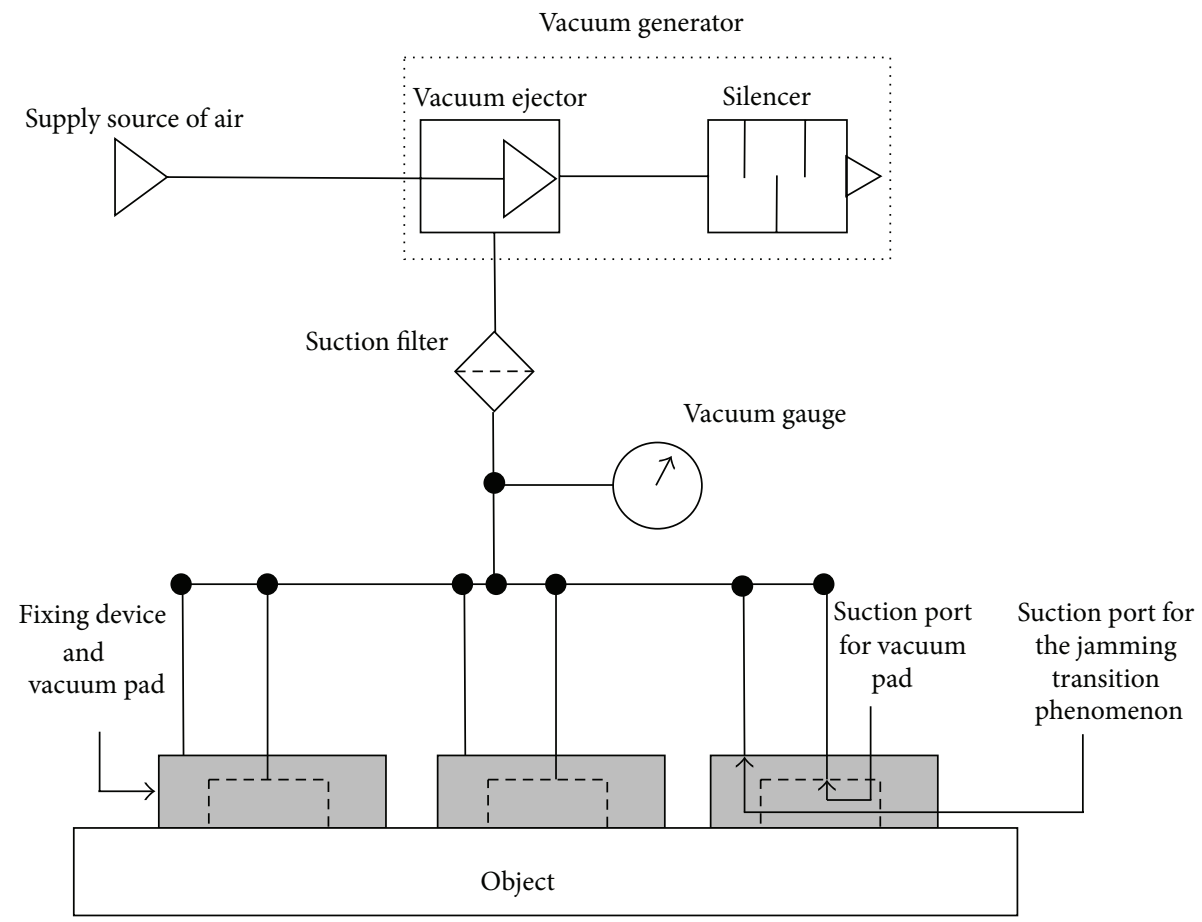

FIgURE 10: Vacuum circuit of the proposed holding device.

\section{Experiment to Evaluate the Performance of the Holding Device}

The holding device is required to contour the target. This ability helps the device to hold the large area of the holding object, and it is evaluated by the degree of adherence. One of the barometers that estimate the degree of adherence is the vacuum degree of the vacuum pad; this is because the adhesion between the vacuum pad of the holding device and the holding object is highly dependent on the air leakage efficiency. The air leakage efficiency directly affects the value of the negative pressure inside the vacuum pad, and the value of the negative pressure is enhanced when the air circulation within the vacuum pad is minimized. As described previously, the sucking force of the proposed device is directly proportional to the negative pressure of the vacuum units, and the performance of the sucking force shows the deformability of the holding device. When the proposed device was affixed to a flat plate, the sucking force was $172 \pm 6$ $\mathrm{N}$; whereas, when it was affixed to a human-shaped phantom (Figure 11), the suction force was $166 \pm 8$-N. The amount of time required to finish holding was 1.6 seconds on average [11].

Next, we evaluated the relationship between the stiffness of our device and the scale of the grains. It has been shown that tetrahedral-shaped grains are better at enhancing the stiffness of the jammed grain aggregate. We fabricated a tetrahedron-shaped grain that has four outshoots. These grains had a tetrapod shape. The grains interlock with neighboring grains and behave as solid-like objects when the density is high. The different-sized grains (Figure 12) were enclosed within the proposed device. Compressive

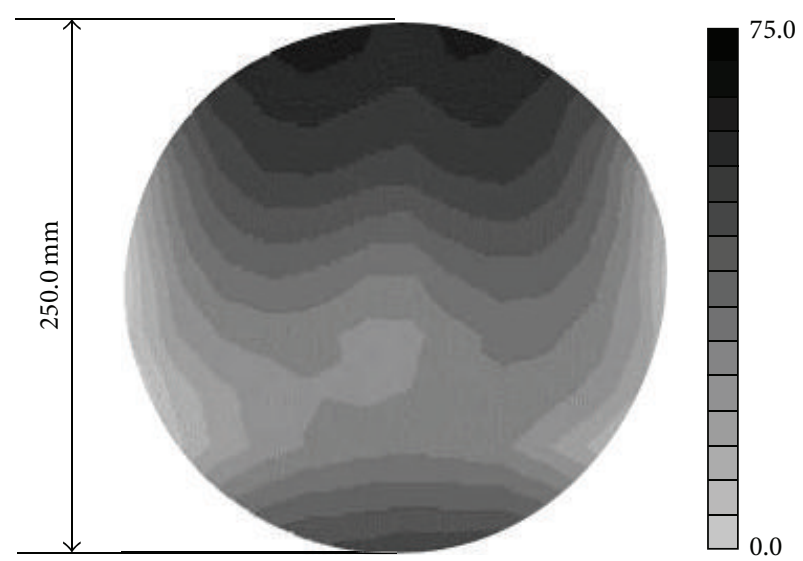

FIGURE 11: Height color map of a curved fixing target.

(downward) and shear (horizontal) loads of 40-N each were applied on the device, which was affixed to a flat plate, and the amount of displacement was measured. Again, 50 trials were conducted.

To evaluate the stiffness of the device in the solid-like phase, the relationship between the deformation of the device and the external load on the device was measured. For the experiment setup, we prepared a flat plate and a curved object. For one of our intended applications-a body-mounted surgical robot-we constructed a human-shaped phantom using the 3D data published by Kawauchi and Mochimaru [14]. The phantom was designed by using the $3 \mathrm{D}$ human shape that was the same as what we used to define the design of the device. The device was installed on the target, and the external 

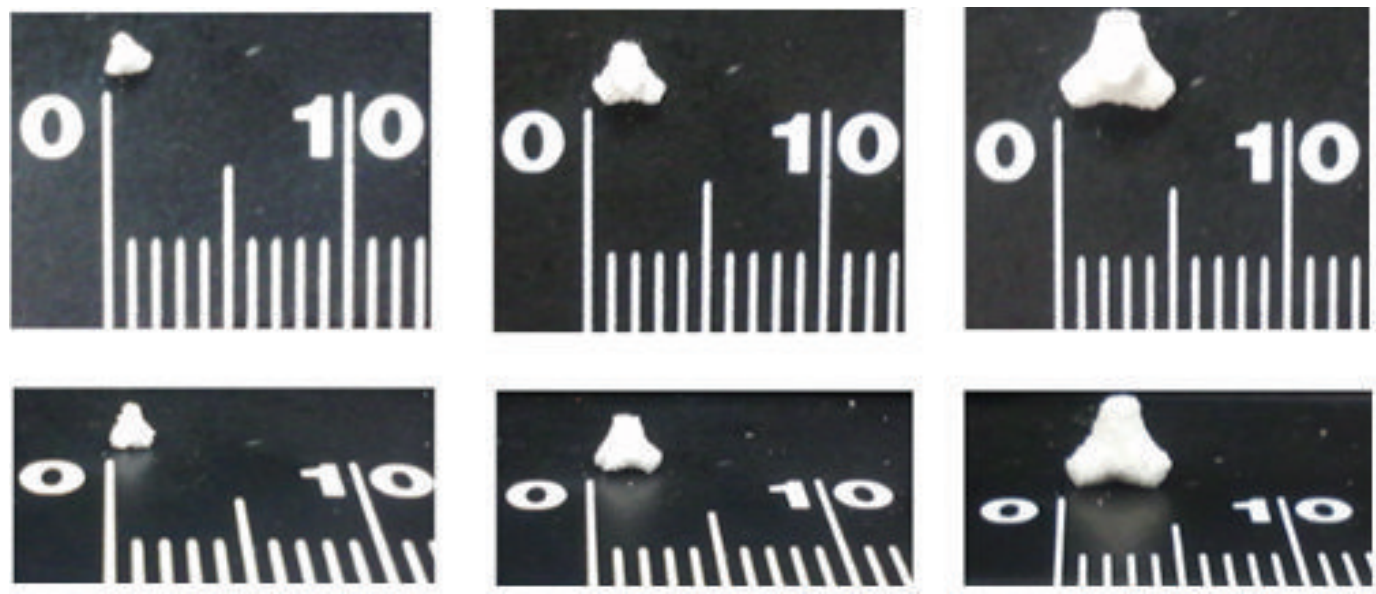

FIGURE 12: Design of grains inside the proposed holding device.
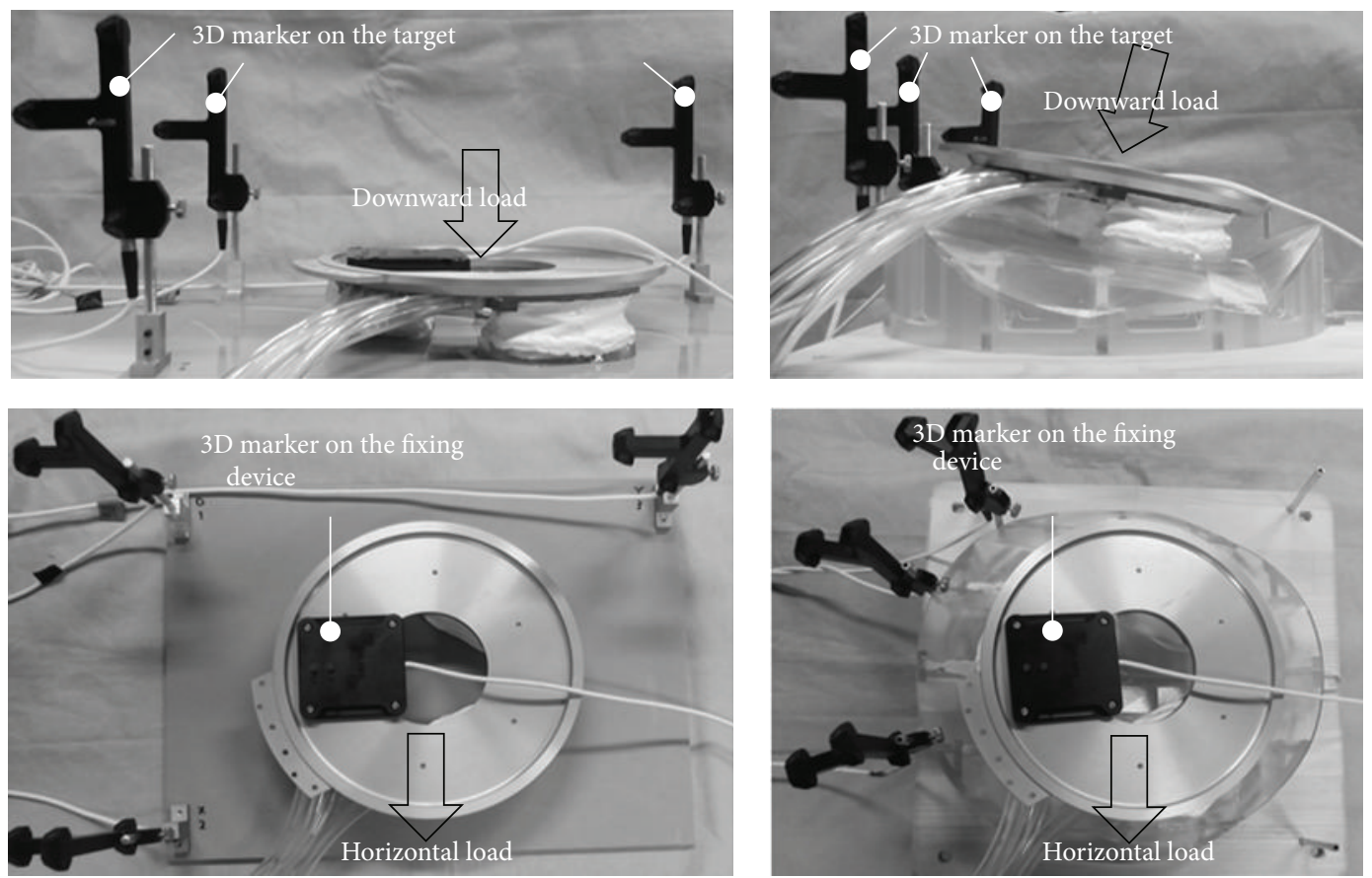

FIGURE 13: Experimental setup to evaluate the stiffness of the holding device.

load was applied. Subsequently, the deformation of the device was evaluated. External downward, horizontal loads of 10, 20, 30,40 , and $50-\mathrm{N}$ were applied to evaluate the deformation of the device. The loads were applied on the top surface of the device. Fifty trials were conducted.

The holding performance of the proposed device was evaluated by the displacement at the top of the device by an external tensile load and upward load. We applied upward load, and the range of force was the same as that of the compressive and shear force; the number of trials was also the same.

To measure the amount of displacement, infrared measurement markers on a $3 \mathrm{D}$ position measurement device (Optotrak, NDI, Canada) were attached to the top of the device and the target (Figures 13 and 14). The amount of deformation was evaluated by measuring the change in the geometrical relationship between the device and the target with 3D markers.

\section{Results of the Holding Device Experiments}

The relationship between the stiffness of the holding device and the size of the grains is shown in Figure 15. The deformation to 40-N downward load significantly differed depending on the size of the grains. Small grains were found to be better for enhancing stiffness.

The displacement of the proposed device due to external load is shown in Figure 16. The size of the grains was $2 \mathrm{~mm}$ in diameter. The displacement for 50-N downward force was found to be $0.08 \mathrm{~mm}$ on average with a standard deviation 

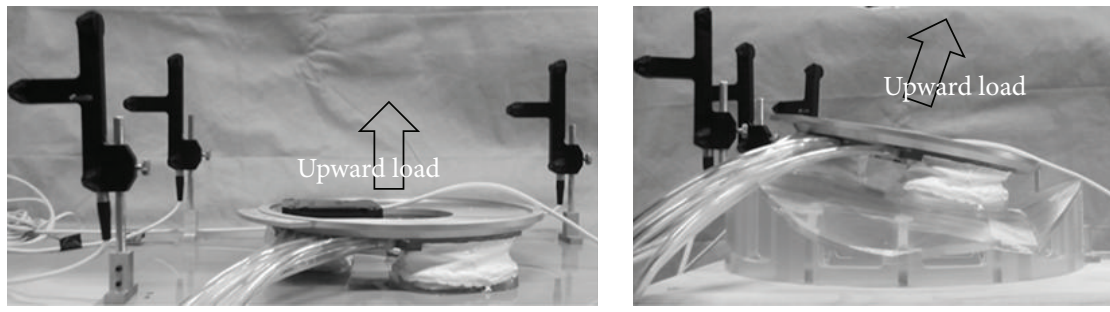

FIGURE 14: Experimental setup to evaluate the performance of the holding device.

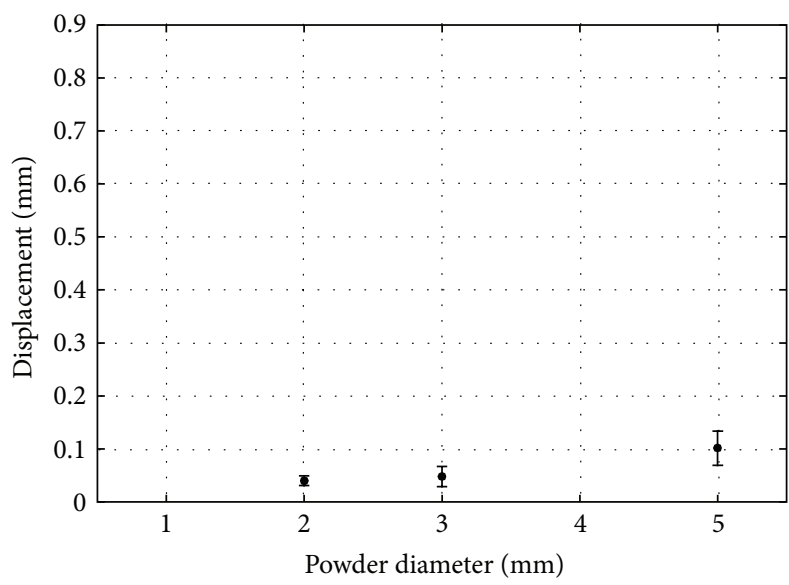

(a)

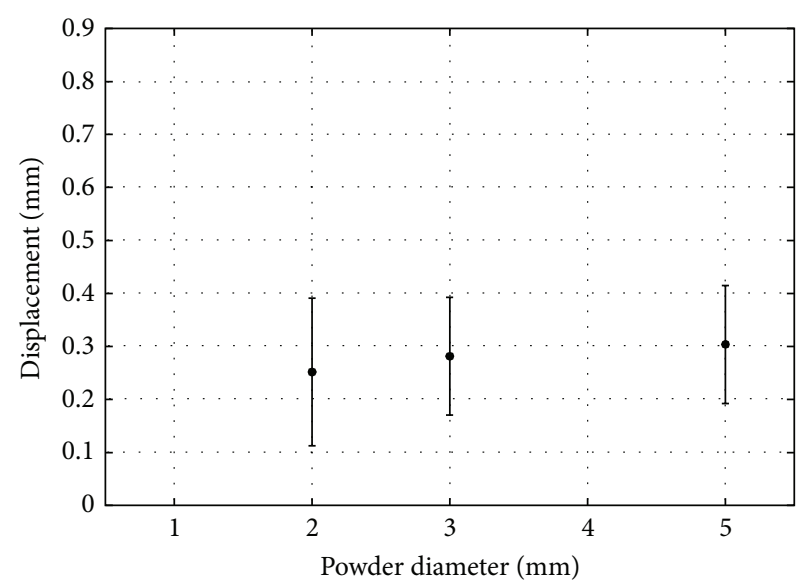

(b)

FIGURE 15: Graph shows the relationship between the grain diameter for the jamming transition and the deformation of the holding device to (a) 40-N downward load, (b) 40-N horizontal load when the holding device is affixed to a flat object.

of $0.02 \mathrm{~mm}$. The displacement for $50-\mathrm{N}$ horizontal force was found to be $0.62 \pm 0.24 \mathrm{~mm}$ when the device was fixed on the plane. When the proposed device was tested on the human-shaped phantom, the displacement was $0.10 \pm$ $0.01 \mathrm{~mm}$ for downward load and $0.35 \pm 0.11 \mathrm{~mm}$ for $50-\mathrm{N}$ horizontal load. The load-displacement relationship on the condition of downward load is close to linear. Thus, when the possible direction of external force is vertical direction, the displacement is specified by the amount of load and easily applicable. However, the load-displacement relationship on the condition of horizontal load is not linear. The plot has a positive slope and is likely to diffuse. The stabilizing effect on the horizontal load is challenging point of this research. Thus, the optimization of grains is important to ensure the stiffness of the fixing device.

The holding performance of the proposed device is shown in Figure 17. The displacement at the top of the device on the flat plate was found to be $0.16 \pm 0.02 \mathrm{~mm}$ for $50-\mathrm{N}$ upward force.

When the holding object was the human-shaped phantom, the displacement was $0.10 \pm 0.01 \mathrm{~mm}$ for an upward load of 50-N. The load-displacement relationship on the condition of upward load is also close to linear and has no problem.

\section{Discussion and Conclusions}

We developed and evaluated a fixing device by utilizing the jamming transition phenomenon and the sucking pad system. To increase the stiffness of the fixing device, we analyzed the effect of the grain shape. We then evaluated the performance of the implemented fixing device on a planar and slightly curved object.

For the improvement of a device that utilizes the jamming transition phenomenon, we tested the relationship between the shape of the grains and the resistance to collapse at the solid-state device that was composed of jammed grain aggregates. The results showed that the spherical grain is not suitable for the proposed fixing device. To achieve high stability in the jammed condition, the tetrahedral shape was effective because the contact force in the normal direction was distributed and the interlock prevented displacement. In addition, the material of grain is also important factor to ensure the stiffness of out proposed fixing device. To use the device repeatedly, toughness is important factor to maintain the nonspherical shape of the particles without becoming warned. In this paper, we used gypseous grain to form the tetrahedral-shaped grain. As a matter of fact, more light and hardened material is adequate for our device. As we have mentioned before, ceramic is one of the adequate materials for our purpose. The evaluation of the material merits further research.

For our purposes, the target shape was planar or curved, and grain flowability was not an obstacle. However, when we use this method for other applications, the behavior of the grains in the fluid-like state and the process of the jamming transition phenomenon could possibly cause problems. 


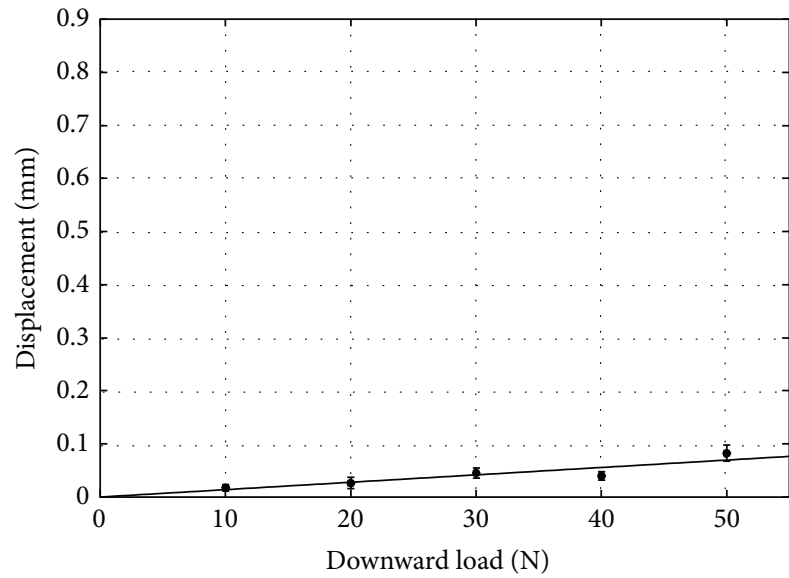

(a)

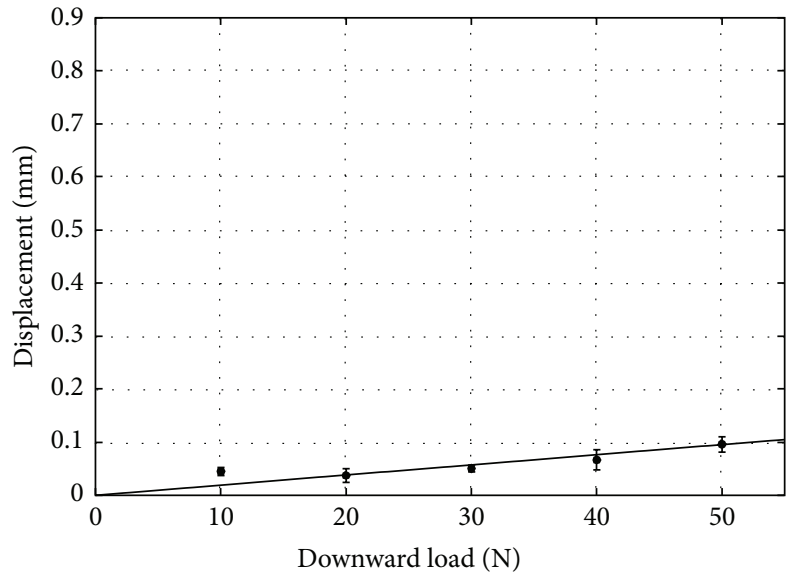

(c)

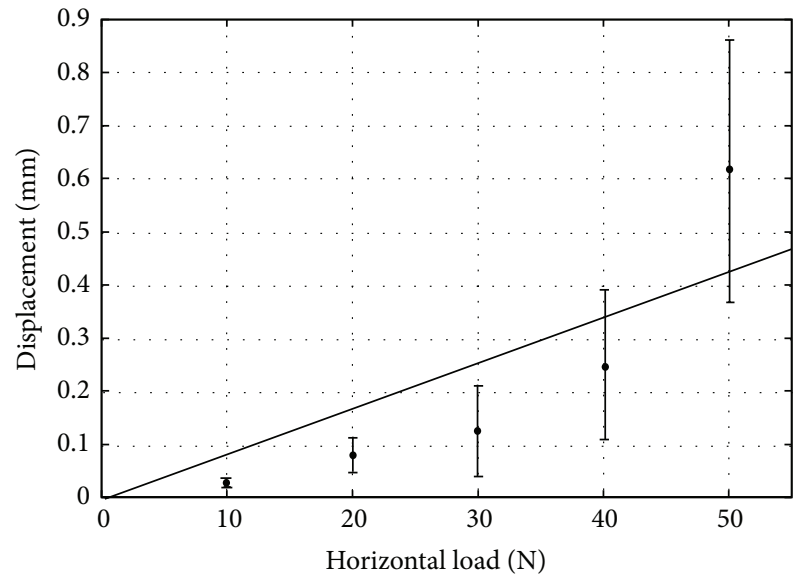

(b)

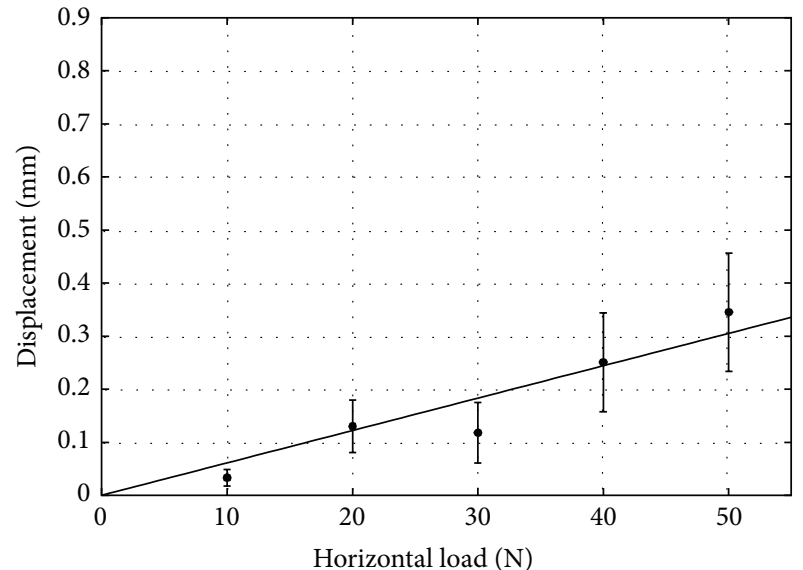

(d)

Figure 16: Compressive and shear load-displacement relationship graph of holding device: (a) displacement to downward force, (b) displacement to horizontal force on flat plate, (c) and (d) graph of displacement to downward and horizontal force on phantom. Solid line is a fitting line that shows the relationship between load and displacement when the device deforms in the elastic deformation range as a real solid object.

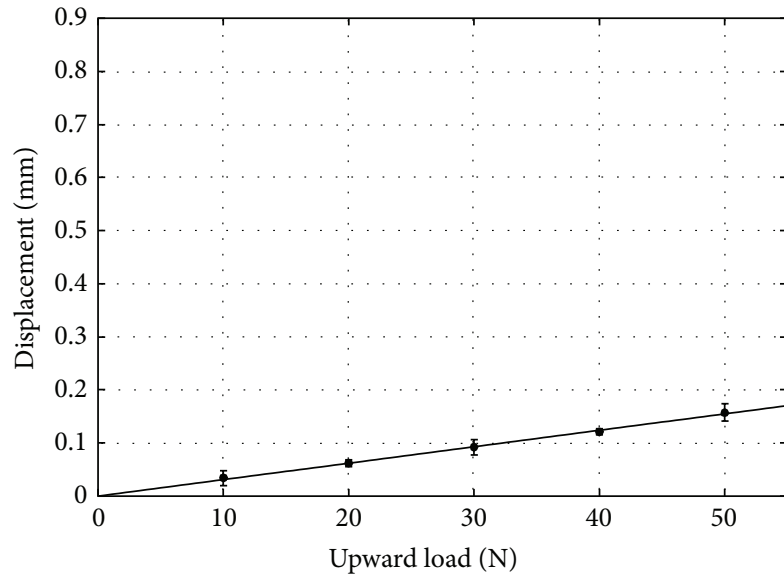

(a)

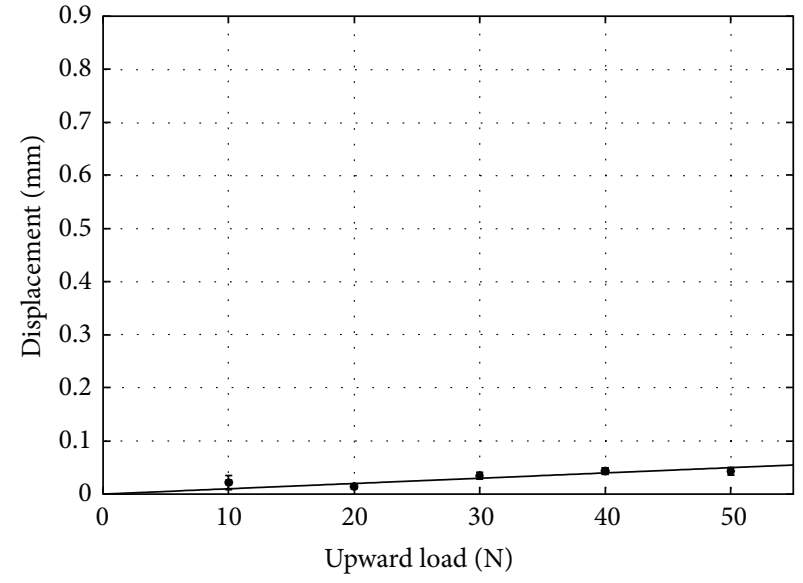

(b)

FIGURE 17: Tensile load-displacement relationship graph of holding device: (a) displacement to upward force on flat plate, (b) graph of displacement to upward force on phantom. Solid line is a fitting line that shows the relationship between load and deformation when the device deforms in the elastic deformation range as a real solid object. 
The effects of these phenomena should be analyzed in future research.

The stability and stiffness of the proposed device on a flat plate and a curved object were evaluated.

In this paper, we evaluated the performance of our device as the fixing device for a small surgical robot that we developed [10]. By the weight of a robot and manual compressing, the device deformed to contour the planer target such as human back. The device gripped the target by using suction force and mechanical constraint by the deformed fixing device. The performance to deform freely and contour the individual shape of the target is ensured by the result that the proposed device is successfully attached onto the curved phantom. The rigidness of the solid-like state that is achieved by the jamming transition phenomenon was also evaluated by the load-deformation relationship.

When an external force was added to the device that was affixed to the target, the change in the geometrical relationship between the device and the target was small. However, the characteristics of the proposed device were not equal to the real solid object. When the proposed device was set on the flat object and a horizontal force was applied, the amount of deformation was large. The displacementhorizontal load curve seemed to be concave upward. We presume that this difference is due to the $3 \mathrm{D}$ conformation of grains in the container.

The vacuum pad performed well in holding the surface of a target and achieved rigid fixation. This result was shown by the tensile load-displacement relationship. It suggests that the combination of the deformable pad, which utilizes the jamming transition phenomenon with adequate grains, and the vacuum pad is an effective approach for holding a planar object.

\section{Conflict of Interests}

The authors declare that there is no conflict of interests regarding the publication of this paper.

\section{References}

[1] W. Townsend, "The BarrettHand grasper-programmably flexible part handling and assembly," Industrial Robot, vol. 27, no. 3, pp. 181-188, 2000.

[2] H. Liu, K. Wu, P. Meusel et al., "Multisensory five-finger dexterous hand: The DLR/HIT hand II," in Proceedings of the IEEE/RSJ International Conference on Intelligent Robots and Systems (IROS '08), pp. 3692-3697, September 2008.

[3] M. Shoham, M. Burman, E. Zehavi, L. Joskowicz, E. Batkilin, and Y. Kunicher, "Bone-mounted miniature robot for surgical procedures: concept and clinical applications," IEEE Transactions on Robotics and Automation, vol. 19, no. 5, pp. 893-901, 2003.

[4] C. J. Walsh, N. C. Hanumara, A. H. Slocum, J.-A. Shepard, and R. Gupta, "A patient-mounted, telerobotic tool for CT-guided percutaneous interventions," Journal of Medical Devices, vol. 2, no. 1, Article ID 011007, 2008.

[5] Z. Qian, Y. Zhao, and Z. Fu, "Development of wall-climbing robots with slipping suction cups," in Proceedings of the 2006
IEEE/RSJ International Conference on Intelligent Robots and Systems, pp. 3417-3422, 2006.

[6] R. L. Tummala, R. Mukherjee, N. Xi et al., "Climbing the walls [robots]," IEEE Robotics \& Automation Magazine, vol. 9, no. 4, pp. 10-19, 2002.

[7] C. Borst, E. W. L. Jansen, C. A. F. Tulleken et al., "Coronary artery bypass grafting without cardiopulmonary bypass and without interruption of native coronary flow using a novel anastomosis site restraining device ('Octopus')," Journal of the American College of Cardiology, vol. 27, no. 6, pp. 1356-1364, 1996.

[8] K. Radermacher, F. Portheine, M. Anton et al., "Computer assisted orthopaedic surgery with image based individual templates," Clinical Orthopaedics and Related Research, no. 354, pp. 28-38, 1998.

[9] E. Brown, N. Rodenberg, J. Amend et al., "Universal robotic gripper based on the jamming of granular material," Proceedings of the National Academy of Sciences of the United States of America, vol. 107, no. 44, pp. 18809-18814, 2010.

[10] A. J. Liu and S. R. Nagel, "Jamming is not just cool any more," Nature, vol. 396, no. 6706, pp. 21-22, 1998.

[11] A. Bekku, J. Kim, Y. Nakajima, and K. Yonenobu, "A bodymounted surgical assistance robot for minimally invasive spinal puncture surgery," in Proceedings of the 5th IEEE RAS \& EMBS International Conference on Biomedical Robotics and Biomechatronics, pp. 19-23, August 2014.

[12] M. Z. Miskin and H. M. Jaeger, "Adapting granular materials through artificial evolution," Nature Materials, vol. 12, no. 4, pp. 326-331, 2013.

[13] S. Koshizuka, A. Nobe, and Y. Oka, "Numerical analysis of breaking waves using the moving particle semi-implicit method," International Journal for Numerical Methods in Fluids, vol. 26, no. 7, pp. 751-769, 1998.

[14] M. Kawauchi and M. Mochimaru, "AIST/HQL measure of human body and shape data base 2003," Tech. Rep. H18PRO503, National Institute of Advanced Industrial Science and Technology, 2006. 

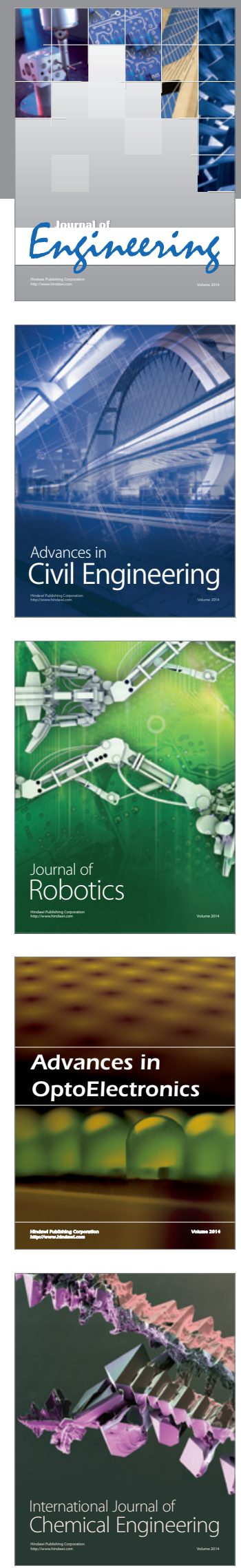

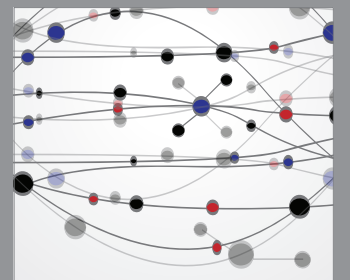

The Scientific World Journal
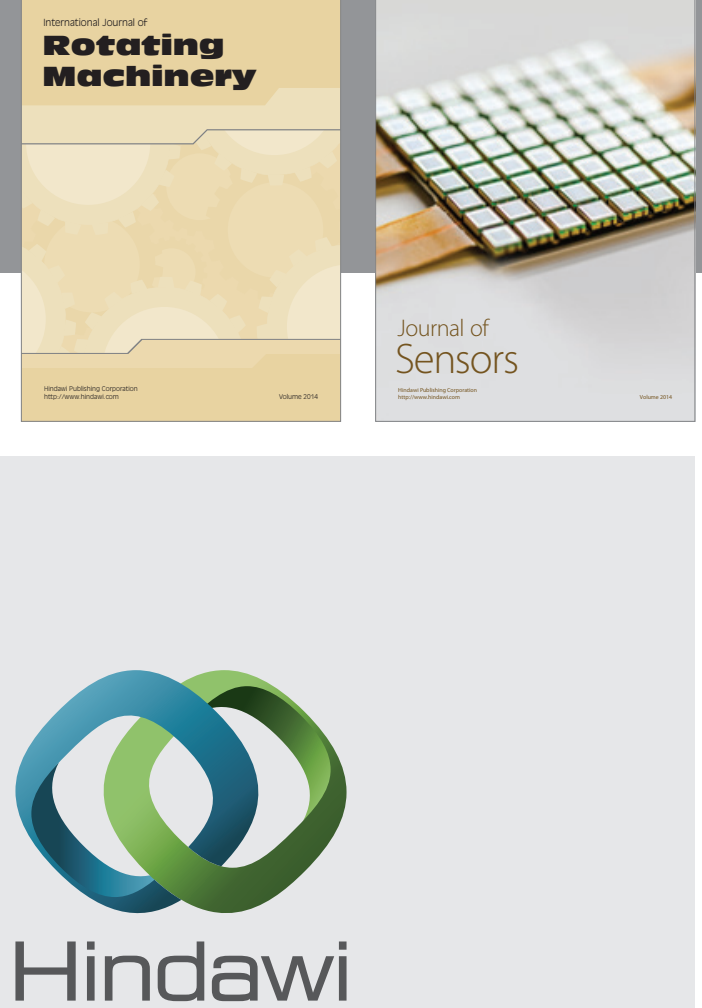

Submit your manuscripts at http://www.hindawi.com
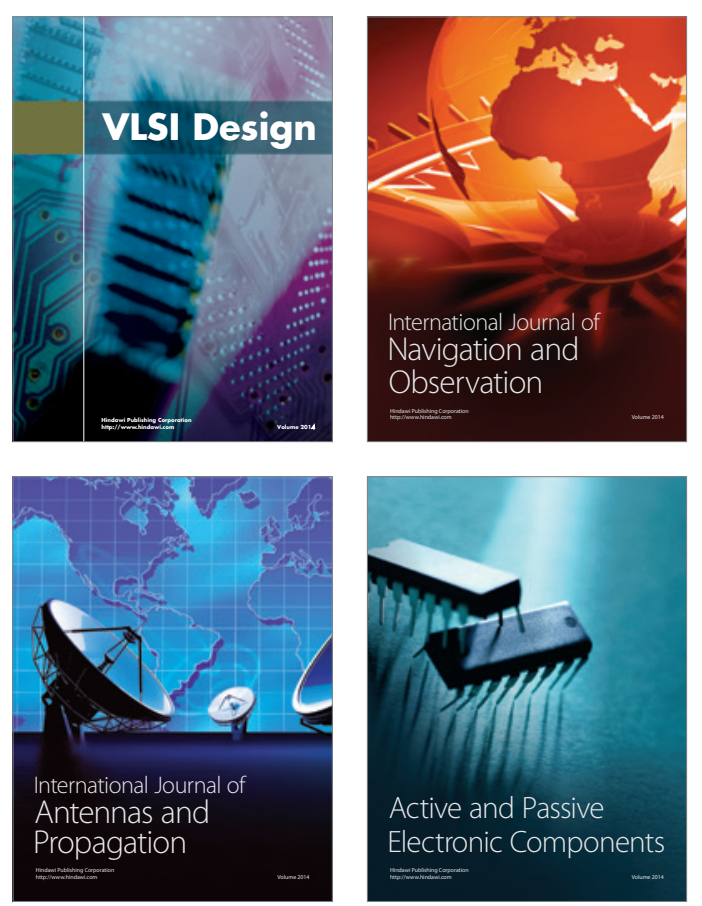
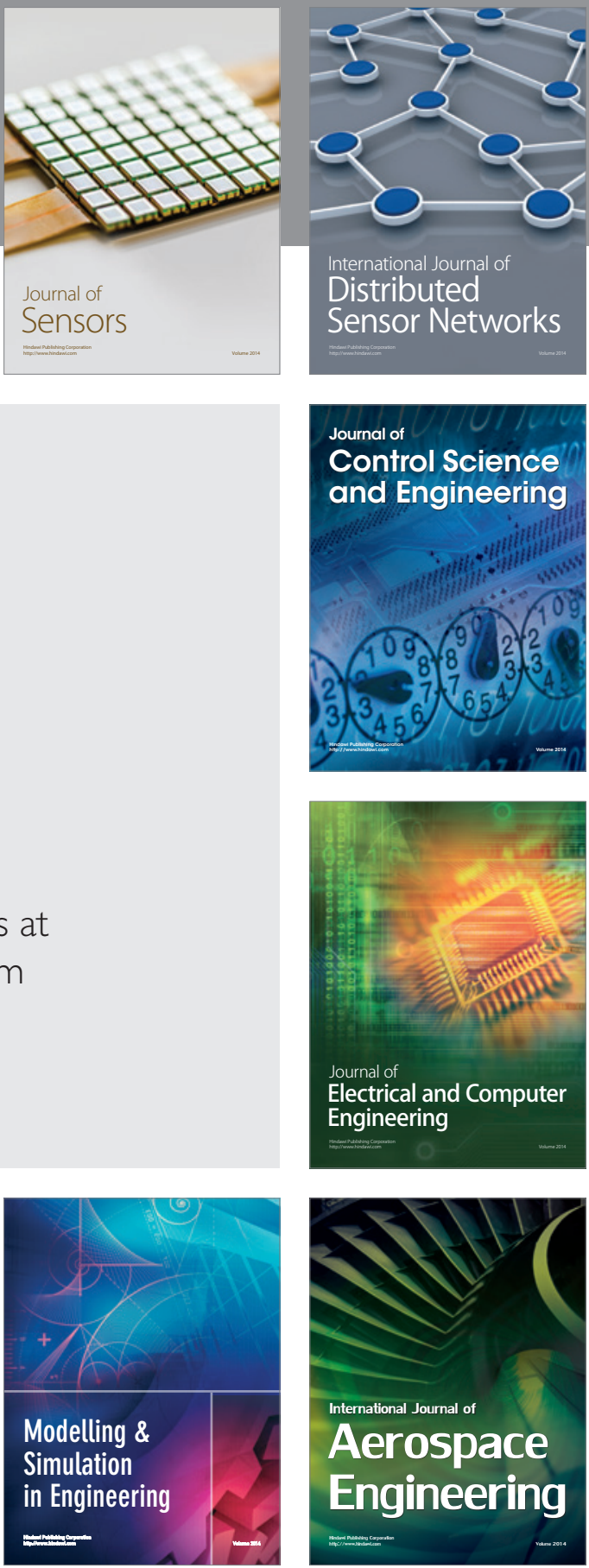

Journal of

Control Science

and Engineering
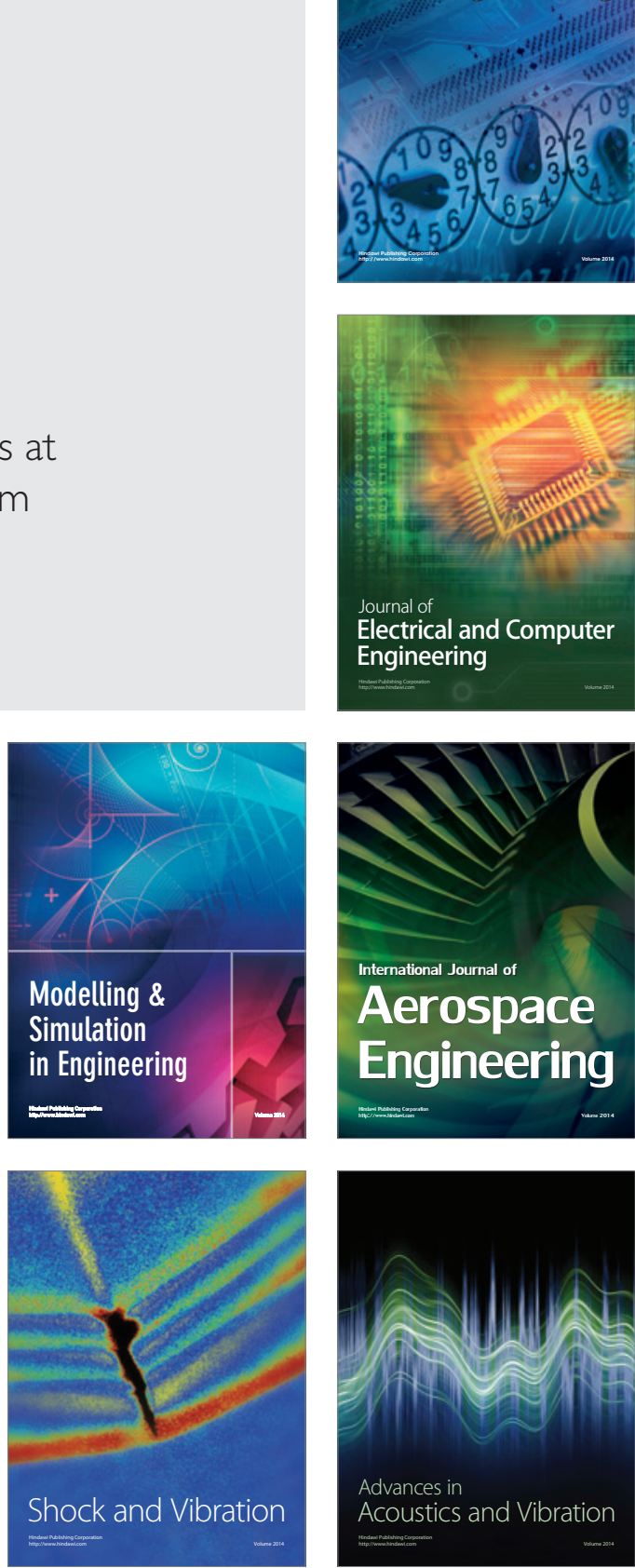